\title{
A single period inventory model for incorporating two-ordering opportunities under imprecise demand information
}

\author{
G. C. Mahata
}

${ }^{a}$ Department of Mathematics, Sitananda College P. O.+ P. S. - Nandigram, Dist. - Purba Medinipur, PIN - 721631, West Bengal, India

\begin{tabular}{l}
\hline A R T I C L E I N F O \\
\hline Article history: \\
Received 20 September 2010 \\
Received in revised form \\
4 December 2010 \\
Accepted 6 December 2010 \\
Available online \\
7 December 2010 \\
\hline Keywords: \\
Single-period inventory \\
Fuzzy demand \\
Reordering strategy \\
Profit maximization
\end{tabular}

A B S T R A C T

\begin{abstract}
The ordering strategy for a single period inventory model is the key to achieve success in the competitive business environment. This article considers demand in a form of fuzzy number and discusses the SPIM in which the retailer has the opportunity to reorder once during the period. The entire period/season is divided into two slots and the reorder is to be made during the mid-season after the early-season demand has been observed. The objective is to find the expected optimal order quantity together with profit maximization. We illustrate the implementation of the proposed model using a numerical example and explain that the explicit consideration of this reordering opportunity could lead us to better results in terms of profitability.
\end{abstract}

(C) 2011 Growing Science Ltd. All rights reserved

\section{Introduction}

Since the development of EOQ model, a lot of research works have been made in the inventory control system. Often uncertainties may be associated with customer demand. So the real-world inventory control problems are imprecisely defined and human interventions are often required to solve these decision-making problems. The single-period inventory control problem is one of these and it has wide applications in the real-world in assisting the decision maker to determine the optimal quantity to order. The classical single period inventory model (SPIM) is a well-known problem. In real business environment, there are various types of SPIM, namely, the stocking of spare parts, perishable items, style goods and special seasonal products, etc., which have a wide relevance in business. Most of the SPIMs describe a business strategy where an item is to be ordered only once to satisfy customer demand for a precise period (Hadley \& Whiten, 1963; Khouja, 1999). Practically, in addition to the supply at the beginning of the sales period, an additional replenishment opportunity exists sometime during the sales period. Moreover, the demand is uncertain, either random or vague, so for multi-product environments like seasonal or fashionable items, there needs a huge initial investment and enormous storage space capacity to avoid loss of good will. Consequently, the * Corresponding author. Tel:

E-mail: gourmahata@yahoo.co.in (G. C. Mahata)

(C) 2011 Growing Science Ltd. All rights reserved. doi: $10.5267 /$ j.ijiec.2010.08.002 
optimal ordering strategy has become a major issue to overcome the organization's limitations and restrictions as well as the customer satisfaction.

This paper focuses on a two ordering strategy throughout the whole season/period where the reorder is to be made during the mid-season after the early-season demand has been observed. Though, the models with such reordering opportunities are very useful in spot selling business, till now, we have not come across any work in this area except the work of Lau and Lau (1997), (1998) and Dutta et al. (2007). Lau and Lau (1997, 1998) developed the model with stochastic demand and divided the whole season into two slots by demand scale and allow shortages for both the slots. Dutta et al. (2007) considered the model with fuzzy demand and divided the whole season into two slots by time scale. They also allowed shortages during slot-1, but it depends on the corresponding profit function to be constructed by the decision maker with his credibility preference to the associated over stock or under stock profit. A similar concept of reordering strategy can be found in Chung and Flynn (2001). They extended the newsboy problem by introducing reactive production, i.e., production occurs in two stages, an anticipatory stage and a reactive stage.

The main purpose of this article is to recast the Dutta et al.'s model (2007) by introducing stochastic variation into the choice of slot-1's demand and reducing shortages during slot-1 as much as possible. Moreover, we aim at providing an uncomplicated reordering-model as compared with Dutta et al. (2007), especially in determining the expected resultant profit. Replenishment rate is considered as instantaneous. As the demands are linguistic in nature and the optimal order quantity in the second slot depends on the demand that arises in the first slot, so the profit function as well as the decision variable during the second slot are also fuzzy quantity. Solution procedure is presented using ordering of fuzzy numbers with respect to their possibilistic mean values (Carlsson \& Fuller, 2001). The objective is to determine a personal policy that will maximize the total resultant profit under the above state of affairs. This paper is organized as follows. In Section 2, some definitions and propositions related to this study are introduced. In Section 3, we redefine the model with reordering opportunity under fuzzy demand and then obtain the optimal order quantities and expected resultant profit step by step to solve the model. Numerical examples are carried out in Section 4 to illustrate the efficiency of the model and finally, Section 5 contains some concluding remarks.

\section{Preliminary concepts}

The demand becomes extremely variable because of shorter product life cycles in the highly competitive market. Hence, the traditional probability theory and statistical method cannot be used properly to describe this kind of uncertainty and the fuzzy theory is employed to deal with these cases. Depending on the manager's judgments or experiences, the uncertainties and imprecision of data are described by linguistic terms such as "the demand is about $d$, not more than $d+\Delta_{2}$ and not less than $d-\Delta_{1}$ ", that is, fuzzy variables. For simplification for the computation, the triangular fuzzy number is employed to describe the fuzzy demand. In this case, the equations for fuzzy total profit will be derived and the numerical analysis will provide us with the management enlightenment.

In order to consider the fuzziness of an inventory problem, we need the following definitions and property relative to this study. Let $X$ be a classical set of objects, called the universe, whose generic elements are denoted by $x$. Membership in a classical sub-set $A$ of $X$ is often viewed as a characteristic function $\mu_{A}$ from $X$ to $\{0,1\}$ such that

$\mu_{A}(x)=\left\{\begin{array}{cc}1 & \text { for } x \in A \\ 0 & \text { otherwise }\end{array}\right.$.

Here $\{0,1\}$ is called a valuation set. If the valuation set is allowed to be the real interval $[0,1], A$ is called a fuzzy set and to distinguish from classical set, it is denoted by $\tilde{A}$. In this case characteristic function $\mu_{A}$ is called membership function of $\tilde{A}$ and is denoted by $\mu_{\tilde{A}}$. The closer the value of $\mu_{\tilde{A}}(x)$ to 1 , the more $x$ belongs to $\tilde{A}$. So a fuzzy set $\tilde{A}$, in the universe of discourse $X$ is completely 
characterized by the set of pairs as $\tilde{A}=\left\{\left(x, \mu_{\tilde{A}}(x)\right), x \in X\right\}$. Clearly $\tilde{A}$ is a subset $X$ that has no sharp boundary and in this case it is normally written as $\widetilde{A} \subseteq X$. A fuzzy set $\tilde{A} \in X$ is said to be normal if there exists at least one $x_{0} \in X$ such that $\mu_{\tilde{A}}\left(x_{0}\right)=1$. A fuzzy set $\widetilde{A} \subseteq X$ is said to be convex if $\forall x_{1} \in X, \forall x_{2} \in X$ and $\lambda \in[0,1]$ we have $\mu_{\tilde{A}}\left(\lambda x_{1}+(1-\lambda) x_{2}\right) \geq \min \left(\mu_{\tilde{A}}\left(x_{1}\right), \mu_{\tilde{A}}\left(x_{2}\right)\right)$.

Any convex normalized fuzzy subset $\tilde{A}$ on the space of real numbers $\mathfrak{R}$ with a continuous membership function $\mu_{\tilde{A}}: \Re \rightarrow[0,1]$ of bounded support is called a fuzzy number (Dubois \& Prade, 1978).

\section{L-R representation of fuzzy numbers (Dubois \& Prade, 1978)}

A fuzzy number $\widetilde{A} \subseteq R$ is said to be a $L-R$ type fuzzy number if its membership function $\mu_{\tilde{A}}$ is given by

$$
\mu_{\tilde{A}}(x)= \begin{cases}L\left(\frac{m-x}{\alpha}\right) & \text { for } x \leq m, \quad \alpha>0 \\ R\left(\frac{x-m}{\beta}\right) & \text { for } x \geq m, \quad \beta>0\end{cases}
$$

where $\mathrm{L}$ is for left and $\mathrm{R}$ is for right reference, $m$ is the mean value of $\tilde{A} . \alpha$ and $\beta$ are called left and right spreads, respectively.

\section{$1.1 \alpha-$ Level set}

$\alpha$-level set (or interval of confidence at level $\alpha$ ) of a fuzzy set $\tilde{A}$ in $X$ is a crisp subset of $X$ denoted by $A(\alpha)$ and is defined by $A(\alpha)=\left\{x \in X / \mu_{\tilde{A}}(x) \geq \alpha\right\} \forall \alpha \in[0,1]$. Let $F$ be the set of all fuzzy numbers. Then for any $\tilde{A}, \tilde{B} \in F$ and for any $\lambda \in R,(\tilde{A} * \tilde{B})(\alpha)=\tilde{A}(\alpha) * \tilde{B}(\alpha),(\lambda \tilde{A})(\alpha)=\lambda \tilde{A}(\alpha)$, where $* \in\{+,-, ., /\}$ and for $*=/, 0 \notin A_{\alpha}$ (Bector $\&$ Chandra, 2005).

\subsection{Triangular fuzzy number (TFN)}

A TFN $\tilde{A}$ is specified by the triplet $(a, b, c)$ and is defined by its continuous membership function $\mu_{\tilde{A}}: \mathfrak{R} \rightarrow[0,1]$ as follows:

$\mu_{\tilde{A}}(x)=\left\{\begin{array}{c}L(x)=\frac{x-a}{b-a} ; a \leq x \leq b, \\ R(x)=\frac{c-x}{c-b} ; \quad b \leq x \leq c, \\ 0 ; \quad \text { otherwise, }\end{array}\right.$

where $a$ is the modal of fuzzy number $\tilde{A}, L: \Re \rightarrow[0,1]$ and $R: \Re \rightarrow[0,1]$ are the left and right shape continuous functions. Hence the closure of the support of $\tilde{A}$ is exactly $[a, c]$.

1.3 Expected mean value of a fuzzy number (Dubois \& Prade, 1978; Liou \& Wang, 1992)

The interval-valued expectation of $\tilde{A}$ is defined as $E(\tilde{A})=\left[E_{*}(\tilde{A}), E^{*}(\tilde{A})\right]$ where $E_{*}(\tilde{A})=$ $\int_{0}^{1} A_{L}(\alpha) d \alpha$ and $E^{*}(\tilde{A})=\int_{0}^{1} A_{R}(\alpha) d \alpha$ are the left and right integral values of $\widetilde{A}$, respectively. The expected mean value of $\tilde{A}$ based on the area measurement index is defined as $\bar{E}(\tilde{A})=\frac{E_{*}(\tilde{A})+E^{*}(\tilde{A})}{2}$.

\subsection{Possibilistic mean value of a fuzzy number}

For a given fuzzy number $\tilde{A}$, the interval valued possibilistic mean is defined as $M(\tilde{A})=\left[M_{*}(\tilde{A}), M^{*}(\tilde{A})\right]$, where $M_{*}(\tilde{A})$ and $M^{*}(\tilde{A})$ are the lower and upper possibilistic mean values of $\tilde{A}$ (Carlsson and Full'er , 2001) and are respectively defined by $M_{*}(\tilde{A})=\frac{\int_{0}^{1} \alpha A_{L}(\alpha) d \alpha}{\int_{0}^{1} \alpha d \alpha}, M^{*}(\tilde{A})=\frac{\int_{0}^{1} \alpha A_{R}(\alpha) d \alpha}{\int_{0}^{1} \alpha d \alpha}$. The 
possibilistic mean value of $\tilde{A}$ is then defined as $\bar{M}(\tilde{A})=\frac{M_{*}(\tilde{A})+M^{*}(\tilde{A})}{2}$. In other words, it can be written as $\bar{M}(\tilde{A})=\int_{0}^{1} \alpha\left(A_{L}(\alpha)+A_{R}(\alpha)\right) d \alpha$. Now, if $\tilde{A}$ and $\tilde{B}$ be two fuzzy numbers, where $A_{\alpha}=\left[A_{\alpha}^{-}, A_{\alpha}^{+}\right]$ and $B_{\alpha}=\left[B_{\alpha}^{-}, B_{\alpha}^{+}\right], \alpha \in[0,1]$, then for ranking fuzzy numbers we have $\tilde{A} \leq \tilde{B} \Leftrightarrow \bar{M}(\tilde{A}) \leq \bar{M}(\tilde{B})$.

\subsection{Graded mean integration value of fuzzy number}

Chen and Hsieh (1999) introduced graded mean integration representation method based on the integral value of graded mean $\alpha$ - level of $L R$-fuzzy number for defuzzifying $L R$-fuzzy numbers. Suppose $\tilde{A}$ is a $L R$-fuzzy number with grade $w$, then according to Chen and Hsieh (1999), graded mean integration representation of $\tilde{A}$ is denoted by $P(\tilde{A})$ and is defined as

$$
P(\tilde{A})=\frac{\int_{0}^{w} \alpha\left(\frac{L^{-1}(\alpha)+R^{-1}(\alpha)}{2}\right) d \alpha}{\int_{0}^{w} \alpha d \alpha}=\int_{0}^{1} \alpha\left(L^{-1}(\alpha)+R^{-1}(\alpha)\right) d \alpha,
$$

with $0<\alpha \leq w$ and $w \leq 1$. Here $L^{-1}$ and $R^{-1}$ are the inverse functions of $L$ and $R$, respectively.

\section{Modeling with reordering opportunity in fuzzy environments}

\subsection{Optimal policy}

In order to consider the SPIM with two ordering strategy under imprecise demand information the following notations are used.

$Q_{1} \quad$ Order quantity at the beginning of the season/period

$Q_{2} \quad$ Order quantity at the beginning of the second slot

$\widetilde{D}_{1} \quad$ Fuzzy demand for slot-1

$\widetilde{D}_{2} \quad$ Fuzzy demand for slot-2

$d_{1} \quad$ Actual realization of demand during slot-1

$d_{2} \quad$ Actual realization of demand during slot-2

$c \quad$ Net purchase cost

$p \quad$ Selling price per unit

$h \quad$ Holding cost per unit for the next season

$s \quad$ Shortage/under stock cost per unit

Suppose the shop-keeper has the opportunity for reorder during the middle of the season. Assuming there is no option for substitution between the products we propose the reordering strategy for an individual item and develop the model for profit maximization. Suppose the demand of the slots is characterized by the triangular fuzzy number $\widetilde{D}_{i}=\left(\underline{D}_{i}, D_{i}, \bar{D}_{i}\right)(i=1,2)$ with the membership function $\mu_{\widetilde{D}_{i}}$, where

$$
\mu_{\widetilde{D}_{i}}(x)= \begin{cases}L_{i}(x)=\frac{x-\underline{D_{i}}}{D_{i}-\underline{D}_{i}} ; & \text { for } \underline{D_{i} \leq \mathrm{x} \leq D_{i}} \\ R_{i}(x)=\frac{\bar{D}_{i}-x}{\bar{D}_{i}-D_{i}} ; & \text { for } D_{i} \leq \mathrm{x} \leq \bar{D}_{i} \\ 0 ; \quad \text { otherwise } & \end{cases}
$$

Looking at the Dutta et al.'s (2007) fuzzy inventory model, the authors divided the whole season into two slots in time scale and considered independent fuzzy demands for both slots. It seems that the authors have solved two single-period problems with leftover items of slot-1 can be used in slot-2, which is unrealistic. Since, such a model is named as 'single-period', therefore, according to the expert's demand information, if $\widetilde{D}=(\underline{D}, D, \bar{D})$ be the total seasonal demand then the demand during slot- 1 should be a certain percent of $\widetilde{D}(\theta \%$ say). Again, most of the time a top manager of an organization depends on the experts opinions about the demand information. So the choice of slot-1's 
demand $\widetilde{D}_{1}$ from the whole seasonal demand $\widetilde{D}$ may not be unique; it may also differ from one expert to another expert. That is, the choice of $\theta$ may vary randomly. Thus, if stochastic variation occurs into the choice of $\widetilde{D}_{1}$, the notion of fuzzy random variable (FRV) must be considered. FRV is a mathematical tool in which both random behavior and fuzzy perception appear simultaneously (Puri \& Ralescu, 1986; Luandjula, 2004). Thus if the expert's opinions about slot-1's demand are described by the phrases " $50 \%$ of $\widetilde{D}$ ", " $60 \%$ of $\widetilde{D}$ ", etc. Then the final choice of slot- 1 's demand can be derived by taking the fuzzy expectation of these fuzzy observations.

If $\widetilde{D}_{1}$ be a discrete FRV such that $P\left(\widetilde{D}_{1}=\tilde{d}_{i}\right)=p_{i}, i=1$ to $n$, then its fuzzy expectation is given by $\widetilde{D}_{1, \exp }=\sum_{i=1}^{n} \tilde{d}_{i} p_{i}$. Therefore, the final choice of $\widetilde{D}_{1}$ is a summarizing fuzzy value of the central tendency of FRV. Consequently, $\widetilde{D}_{1}=\left(\underline{D}_{1}, D_{1}, \bar{D}_{1}\right)$ becomes a fuzzy quantity.

Further, Dutta et al. (2007) allowed shortages during slot-1. But, in a competitive market, if shortages occur during the mid-season of the period and customers are going back without fulfillment of their demand, it not only affects the expected profit in the first slot but also it changes the demand rate of the next slot. To overcome the loss of goodwill, without loss of generality it can be assumed that the maximum possible demand during slot- 1 is $\widetilde{D}_{1}$ with the lowest membership grade zero. Thus if the decision-maker (DM) chooses $Q_{1}^{*}=\bar{D}_{1}$ then the possibility of shortages during slot-1 is nil rather there is obvious excess after the end of this slot. In this case, the DM is not bothering about the certain excess of products during slot- 1 rather he gives the priority to customer satisfaction during the middle of the season. In addition, as replenishment is instantaneous, shortages may be backordered, if any. Therefore, if $Q_{1}^{+}$be the number of quantities left after the end of slot-1, then $Q_{1}^{+}$is defined as $Q_{1}^{+}=Q_{1}^{*}-d_{1}$, where $d_{1}$ is the actual occurrence of demand in slot-1. Let $Q_{2}^{+}$be the optimal order quantity to be needed for maximizing the profit function in the second slot individually based on the fuzzy demand $\widetilde{D}_{2}$, if no quantities are supplied from slot- 1 . Then the optimal order quantity for the second slot at the beginning of slot- 2 is defined as follows:

$Q_{2}^{o p t}=\left\{\begin{array}{ll}0 & \text { for } Q_{1}^{+} \geq Q_{2}^{*} \\ Q_{2}^{*}-Q_{1}^{+} & \text {for } 0 \leq Q_{1}^{+} \leq Q_{2}^{*}\end{array}\right.$.

Therefore, our task is to find out the only decision variables $Q_{2}^{*}$ that maximizes the total profit function for the second slot.

\subsection{Determination of optimal order quantity $Q_{2}^{*}$}

As demand $\widetilde{D}_{2}$ in slot-2 is imprecisely prescribed, it causes a fuzzy over stock profit $(\widetilde{O P})$ and fuzzy under stock profit $(\widetilde{U P})$ and hence the resultant profit function also becomes a fuzzy quantity $\tilde{P}\left(Q_{2}, \widetilde{D}_{2}\right)$ (say). If $d_{2}$ be the actual demand realization of fuzzy demand $\widetilde{D}_{2}$ in slot -2 , then for this slot the profit function can be formulated as

$P\left(Q_{2}, d_{2}\right)=\left\{\begin{array}{lll}O P=(p+h) d_{2}-(c+h) Q_{2} & \text { for } d_{2} \leq Q_{2} \\ U P=(p-c+s) Q_{2}-s d_{2} & \text { for } d_{2}>Q_{2}\end{array}\right.$

Therefore the fuzzy overstock and fuzzy under stock profit functions are respectively given by

$\widetilde{O P}\left(Q_{2}, \widetilde{D}_{1}\right)=(p+h) \widetilde{D}_{2}-(c+h) Q_{2}$ along with the membership function

$\mu_{\widetilde{O P}}(\gamma)=\left\{\begin{array}{ccc}\sup _{\gamma=(p+h) d_{2}-(c+h) Q_{2}}\left\{\mu_{\widetilde{D_{2}}}\left(d_{2}\right)\right\} & \text { for } & d_{2} \leq Q_{2} \\ 0 & \text { for } & d_{2}>Q_{2}\end{array}\right.$

and

$\widetilde{U P}\left(Q_{2}, \widetilde{D}_{1}\right)=(p-c+s) Q_{2}-s \widetilde{D}_{2}$ 
along with the membership function

$$
\mu_{\widetilde{U P}}(\gamma)=\left\{\begin{array}{cc}
\sup _{\gamma=(p-c+s) Q_{2}-s d_{2}}\left\{\mu_{\widetilde{D_{2}}}\left(d_{2}\right)\right\} & \text { for } d_{2} \geq Q_{2} \\
0 & \text { for } d_{2}<Q_{2}
\end{array} .\right.
$$

Without loss of generality, the resultant fuzzy profit function $\widetilde{P}\left(Q_{2}, \widetilde{D}_{2}\right)$ can be formulated as $\tilde{P}\left(Q_{2}, \widetilde{D}_{2}\right)=\widetilde{O P}\left(Q_{2}, \widetilde{D}_{1}\right) \cup \widetilde{U P}\left(Q_{2}, \widetilde{D}_{1}\right)$ with the membership function

$\mu_{\tilde{P}}(\gamma)=\max \left\{\mu_{\widetilde{O P}}(\gamma), \mu_{\widetilde{U P}}(\gamma)\right\}$. Since $\widetilde{P}\left(Q_{2}, \widetilde{D}_{2}\right)$ is a fuzzy quantity and so it can be directly maximized. Now using several $\alpha$-level set and possibilistic mean value method, one can find out the required $Q_{2}^{*}$ by maximizing the mean or expected value of this fuzzy profit function. If $P\left(Q_{2}, \alpha\right)$ be the $\alpha$-level set of $\tilde{P}\left(Q_{2}, \widetilde{D}_{2}\right)$, then it can be derived as follows:

$$
\begin{aligned}
P\left(Q_{2}, \alpha\right) & =O P\left(Q_{2}, \alpha\right) \cup U P\left(Q_{2}, \alpha\right)=\left[P_{L}\left(Q_{2}, \alpha\right), P_{R}\left(Q_{2}, \alpha\right)\right] \\
& =\left[\min \left\{O P_{L}\left(Q_{2}, \alpha\right), U P_{L}\left(Q_{2}, \alpha\right)\right\}, \max \left\{O P_{R}\left(Q_{2}, \alpha\right), U P_{R}\left(Q_{2}, \alpha\right)\right\}\right] .
\end{aligned}
$$

Thus the possibilistic mean value of fuzzy profit function $\tilde{P}\left(Q_{2}, \widetilde{D}_{2}\right)$ is given by

$\bar{M}(\tilde{P})=\int_{0}^{1} \alpha\left(P_{L}(\alpha)+P_{R}(\alpha)\right) d \alpha$.

For this we need to know the $\alpha$-cut of the fuzzy profit function $\widetilde{P}\left(Q_{2}, \widetilde{D}_{2}\right)$ for all $\alpha \in[0,1]$. Details analysis of deriving $P\left(Q_{2}, \alpha\right)$ can be found in Dutta et al. (2007). In order to determine the optimal $Q_{2}^{*}$, in this context, we develop the following features about $\bar{M}(\tilde{P})$. Let us first define $D_{2,0}=\frac{(p+h) \underline{D}_{2}+s \bar{D}_{2}}{p+s+h}$, then it is easy to identify the position of $Q_{2}^{*}$ in $\left[\underline{D}_{2}, \bar{D}_{2}\right]$. We then have two cases, namely, $D_{2,0} \leq D_{2}$ or $>D_{2}$ or equivalently, $s r_{2}-l_{2}(p+h) \leq 0$ or $>0$, where $l_{2}$ and $r_{2}$ are the left and right spreads of $\widetilde{D}_{2}$, respectively.

Case 3.2.a. When $s r_{2}-l_{2}(p+h) \leq 0$ then $Q_{2}^{*} \in\left[D_{2,0}, D_{2}\right]$ and then the possibilistic mean value $\bar{M}(\tilde{P})$ of fuzzy profit function $\widetilde{P}\left(Q_{2}, \widetilde{D}_{2}\right)$ is obtained as

$$
\begin{aligned}
\bar{M}(\tilde{P})= & (p-c+s) Q_{2}-\frac{1}{2}\left[(p+s+h) \alpha_{2}^{2}+s\left\{L_{2}\left(Q_{2}\right)\right\}^{2}\right] Q_{2}+ \\
& (p+h) \int_{0}^{\alpha_{2}} \alpha D_{2, L}(\alpha) d \alpha-s \int_{\alpha_{2}}^{1} \alpha D_{2, R}(\alpha) d \alpha-s \int_{L_{2}\left(Q_{2}\right)}^{1} \alpha D_{2, L}(\alpha) d \alpha
\end{aligned}
$$

where $\alpha_{2}=\frac{(p-c+s) Q_{2}-\left\{(p+h) \underline{D}_{2}+s \underline{D}_{2}\right\}}{(p+h) l_{2}-s r_{2}}<L_{2}\left(Q_{2}\right)$. Therefore the optimal value of order quantity $Q_{2}^{*}$ is obtained by setting the first derivative of $\bar{M}(\tilde{P})$ with respect to $Q_{2}$ equal to zero. That is,

$\frac{\partial \bar{M}\left(Q_{2}^{*}\right)}{\partial Q_{2}} \equiv(p-c+s) Q_{2}-\frac{1}{2}\left[(p+s+h) \alpha_{2}^{2}+s\left\{L_{2}\left(Q_{2}^{*}\right)\right\}\right]=0$

subject to the condition $p-2 c-h \leq 0$.

Again, if $p-2 c-h>0$, then the optimal $Q_{2}^{*}$ lies between $D_{2}$ and $\bar{D}_{2}$. In this case, the possibilistic mean value $\bar{M}(\widetilde{P})$ of the fuzzy profit function $\widetilde{P}\left(Q_{2}, \widetilde{D}_{2}\right)$ is given by 


$$
\begin{aligned}
\bar{M}(\tilde{P})=-(c+h) Q_{2}+ & \frac{1}{2}\left[(p+h)\left\{R_{2}\left(Q_{2}\right)\right\}^{2}\right] Q_{2} \\
& +(p+h)\left[\int_{0}^{1} \alpha D_{2, L}(\alpha) d \alpha+\int_{R_{2}\left(Q_{2}\right)}^{1} \alpha D_{2, R}(\alpha) d \alpha\right.
\end{aligned}
$$

subject to the following condition

$$
\frac{\partial \bar{M}\left(Q_{2}^{*}\right)}{\partial Q_{2}} \equiv-(c+h) Q_{2}+\frac{1}{2}\left[(p+h)\left\{R_{2}\left(Q_{2}^{*}\right)\right\}^{2}\right]=0 .
$$

Case 3.2.b. When $s r_{2}-l_{2}(p+h)>0$ or $D_{2,0}>D_{2}$, then $Q_{2}^{*} \in\left[D_{2}, D_{2,0}\right]$. In this case, the possibilistic mean value $\bar{M}(\tilde{P})$ of fuzzy profit function $\widetilde{P}\left(Q_{2}, \widetilde{D}_{2}\right)$ is given by

$$
\begin{aligned}
\bar{M}(\tilde{P})= & -(c+h) Q_{2}+\frac{1}{2}\left[(p+s+h) \alpha_{2}^{2}+(p+h)\left\{R_{2}\left(Q_{2}\right)\right\}^{2}\right] Q_{2} \\
& -\mathrm{s} \int_{0}^{\alpha_{2}} \alpha D_{2, R}(\alpha) d \alpha+(p+h)\left[\int_{\alpha_{2}}^{1} \alpha D_{2, L}(\alpha) d \alpha+\int_{R_{2}\left(Q_{2}\right)}^{1} \alpha D_{2, R}(\alpha) d \alpha,\right.
\end{aligned}
$$

along with the relation

$\frac{\partial \bar{M}\left(Q_{2}^{*}\right)}{\partial Q_{2}} \equiv(p-c+s) Q_{2}-\frac{1}{2}\left[(p+s+h) \alpha_{2}^{2}+s\left\{L_{2}\left(Q_{2}^{*}\right)\right\}\right]=0$

subject to the condition $-(c+h)+(p+h)\left(\frac{\bar{D}_{2}-D_{2,0}}{\bar{D}_{2}-D_{2}}\right)^{2} \leq 0$. Otherwise, $Q_{2}^{*}$ lies between $D_{2,0}$ and $\bar{D}_{2}$ and $Q_{2}^{*}$ can be computed as defined either in Eq. (7) or in Eq. (9).

\subsection{Total expected optimal order quantity}

In this subsection, we find out the total expected order quantity for the whole season. To calculate the total expected order quantity $Q_{2}^{o p t}$, the optimal policy defined in Eq. (1) can be written as

$$
Q_{2}^{\text {opt }}=\left\{\begin{array}{cl}
0 & \text { for } \underline{D}_{1} \leq d_{1} \leq Q_{1}^{*}-Q_{2}^{*}, \\
Q_{2}^{*}-Q_{1}^{+} & \text {for } \max \left\{\underline{D}_{1}, Q_{1}^{*}-Q_{2}^{*}\right\}<d_{1} \leq \bar{D}_{1} .
\end{array}\right.
$$

This is a fuzzy quantity as demand $\widetilde{D}_{1}$ is a fuzzy quantity, so uncertainty occurs in case $Q_{2}^{o p t}$ too. Thus, the expected value of $Q_{2}^{o p t}$ can be predict as

$\bar{M}\left(\tilde{Q}_{2}^{o p t}\right)=\int_{0}^{1} \alpha\left(Q_{2, L}^{o p t}(\alpha)+Q_{2, R}^{o p t}(\alpha)\right) d \alpha$,

where $Q_{2}^{o p t}(\alpha)=\left[Q_{2, L}^{o p t}(\alpha), Q_{2, R}^{o p t}(\alpha)\right]$ and is obtained as follows.

Condition - 3.3.a. When $Q_{1}^{*}-Q_{2}^{*}<\underline{D}_{1}$ then

(i) If $Q_{1}^{*}-Q_{2}^{*} \leq D_{1}$ then

$$
Q_{2}^{o p t}= \begin{cases}{\left[0, Q_{2}^{*}-Q_{1}^{*}+D_{1, R}(\alpha)\right]} & \text { for } \alpha \leq L_{1}\left(Q_{1}^{*}-Q_{2}^{*}\right) \\ {\left[Q_{2}^{*}-Q_{1}^{*}+D_{1, L}(\alpha), Q_{2}^{*}-Q_{1}^{*}+D_{1, R}(\alpha)\right]} & \text { for } \alpha>L_{1}\left(Q_{1}^{*}-Q_{2}^{*}\right) .\end{cases}
$$

(ii) If $Q_{1}^{*}-Q_{2}^{*}>D_{1}$ then 
$Q_{2}^{o p t}= \begin{cases}{\left[0, Q_{2}^{*}-Q_{1}^{*}+D_{1, R}(\alpha)\right]} & \text { for } \alpha \leq R_{1}\left(Q_{1}^{*}-Q_{2}^{*}\right) \\ {[0,0]} & \text { for } \alpha>R_{1}\left(Q_{1}^{*}-Q_{2}^{*}\right) .\end{cases}$

Therefore, the total expected optimal order quantity combining slot- 1 and slot- 2 is calculated by

$Q_{1}^{*}+$ Expected $Q_{2}^{o p t}=\bar{D}_{1}+\bar{M}\left(\widetilde{Q}_{2}^{o p t}\right)$.

\subsection{Total expected resultant profit}

In order to calculate the expected value of total resultant profit $(T R P)$, it is required to construct the individual resultant profit functions according to the optimal policy (3).

Case - 3.4.a. When $Q_{1}^{+} \geq Q_{2}^{*}$, then we have $Q_{2}^{o p t}=0$ and the expected value of TRP is given by

$T R P= \begin{cases}p d_{1}-c Q_{1}^{*}+(p+h) d_{2}-h Q_{1}^{+} & \text {for } d_{2} \leq Q_{1}^{+} \\ p d_{1}-c Q_{1}^{*}+(p+s) Q_{1}^{+}-s d_{2} & \text { for } d_{2} \leq Q_{1}^{+}\end{cases}$

Case - 3.4.b. When $Q_{1}^{+} \geq 0$ and $Q_{1}^{+} \leq Q_{2}^{*}$, then $Q_{2}^{o p t}=Q_{2}^{*}-Q_{1}^{+}$and the expected value of TRP is obtained as

$T R P= \begin{cases}(p-c) d_{1}+(p+h) d_{2}-(c+h) Q_{2}^{*} & \text { for } d_{2} \leq Q_{2}^{*} \\ (p-c) d_{1}+(p-c+s) Q_{2}^{*}-s d_{2} & \text { for } d_{2}>Q_{2}^{*}\end{cases}$

Obviously, this total resultant profit is a fuzzy quantity, $\widetilde{T R P}$ (say). Combining above two individual profits we can find out the expected or mean value of this fuzzy profit function. Thus, if $\operatorname{TRP}(\alpha)=$ $\left[T R P_{L}(\alpha), T R P_{R}(\alpha)\right]$ be the $\alpha-$ level set of this fuzzy resultant profit function, then its possibilistic mean value is given by

$\bar{M}(\widetilde{T R P})=\int_{0}^{1} \alpha\left(T R P_{L}(\alpha)+T R P_{R}(\alpha)\right) d \alpha$.

When $Q_{1}^{*}-Q_{2}^{*}<\underline{D}_{1}$ then case-2.4.a will never happen and we have

$\operatorname{TRP}(\alpha)=\left[(p-c) D_{1, L}(\alpha)+\bar{M}(\tilde{P}),(p-c) D_{1, R}(\alpha)+\bar{M}(\tilde{P})\right]$ for all $\alpha \in[0,1]$.

Again, when $Q_{1}^{*}-Q_{2}^{*}>\underline{D}_{1}$ then $\operatorname{TRP}(\alpha)$ is given as follows:

If $Q_{1}^{*}-Q_{2}^{*} \leq D_{1}$ then

$\operatorname{TRP}(\alpha)$

$= \begin{cases}{\left[\begin{array}{cc}\min \left\{p D_{1, L}(\alpha)-c Q_{1}^{*}+\bar{M}\left(\tilde{P}_{d}\right),(p-c)\left(Q_{1}^{*}-Q_{2}^{*}\right)+\bar{M}(\tilde{P})\right\}, \\ \max \left\{p\left(Q_{1}^{*}-Q_{2}^{*}\right)-c Q_{1}^{*}+\bar{M}\left(\tilde{P}_{d}\right),(p-c) D_{1, R}(\alpha)+\bar{M}(\tilde{P})\right\}\end{array}\right],} & \text { for } \alpha \leq L_{1}\left(Q_{1}^{*}-Q_{2}^{*}\right), \\ {\left[p D_{1, L}(\alpha)-c Q_{1}^{*}+\bar{M}(\tilde{P}), p D_{1, R}(\alpha)-c Q_{1}^{*}+\bar{M}(\tilde{P})\right],} & \text { for } \alpha>L_{1}\left(Q_{1}^{*}-Q_{2}^{*}\right)\end{cases}$

If $Q_{1}^{*}-Q_{2}^{*}>D_{1}$ then

$\operatorname{TRP}(\alpha)$

$= \begin{cases}{\left[\begin{array}{c}\min \left\{p D_{1, L}(\alpha)-c Q_{1}^{*}+\bar{M}\left(\tilde{P}_{d}\right),(p-c)\left(Q_{1}^{*}-Q_{2}^{*}\right)+\bar{M}(\tilde{P})\right\}, \\ \max \left\{p\left(Q_{1}^{*}-Q_{2}^{*}\right)-c Q_{1}^{*}+\bar{M}\left(\tilde{P}_{d}\right),(p-c) D_{1, R}(\alpha)+\bar{M}(\tilde{P})\right\}\end{array}\right],} & \text { for } \alpha \leq R_{1}\left(Q_{1}^{*}-Q_{2}^{*}\right), \\ {\left[p D_{1, L}(\alpha)-c Q_{1}^{*}+\bar{M}(\tilde{P}), p D_{1, R}(\alpha)-c Q_{1}^{*}+\bar{M}(\tilde{P})\right],} & \text { for } \alpha>R_{1}\left(Q_{1}^{*}-Q_{2}^{*}\right)\end{cases}$

where $\bar{M}(\tilde{P})$ is the possibilistic mean of the dummy profit function $P_{d}\left(G\left(Q_{1}^{+}\right)\right)$and is determined from either of the Eqs. (4), (6) or Eq. (8) by replacing $c=0$ and $Q_{1}^{*}=G\left(Q_{1}^{+}\right)$according to their 
subsequent conditions, respectively. In this case, $G\left(Q_{1}^{+}\right)$denotes the graded mean of $Q_{1}^{+}$and is determined as follows:

If $Q_{1}^{*}-Q_{2}^{*} \leq D_{1}$ then $Q_{1}^{+}(\alpha)=\left[Q_{2}^{*}, Q_{1}^{*}-D_{1, L}(\alpha)\right]$ for $\alpha \leq L\left(Q_{1}^{*}-Q_{2}^{*}\right)$.

Thus, $G\left(Q_{1}^{+}\right)=\frac{\int_{0}^{L\left(Q_{1}^{*}-Q_{2}^{*}\right)} \alpha\left(\frac{Q_{2}^{*}+Q_{1}^{*}-D_{1, L}(\alpha)}{2}\right) d \alpha}{\int_{0}^{L\left(Q_{1}^{*}-Q_{2}^{*}\right)} \alpha d \alpha}$.

Again, if $Q_{1}^{*}-Q_{2}^{*}>D_{1}$ then $Q_{1}^{+}(\alpha)= \begin{cases}{\left[Q_{2}^{*}, Q_{1}^{*}-D_{1, L}(\alpha)\right]} & \text { for } \alpha \leq R\left(Q_{1}^{*}-Q_{2}^{*}\right) \\ {\left[Q_{1}^{*}-D_{1, R}(\alpha), Q_{1}^{*}-D_{1, L}(\alpha)\right]} & \text { for } \alpha>R\left(Q_{1}^{*}-Q_{2}^{*}\right) .\end{cases}$

Hence,

$G\left(Q_{1}^{+}\right)=\frac{\int_{0}^{R\left(Q_{1}^{*}-Q_{2}^{*}\right)} \alpha\left(\frac{Q_{2}^{*}+Q_{1}^{*}-D_{1, L}(\alpha)}{2}\right) d \alpha+\int_{R\left(Q_{1}^{*}-Q_{2}^{*}\right)}^{1} \alpha\left(\frac{Q_{1}^{*}-D_{1, R}(\alpha)+Q_{1}^{*}-D_{1, L}(\alpha)}{2}\right) d \alpha}{\int_{0}^{1} \alpha d \alpha}$.

Thus, the expected total resultant profit, $\bar{M}(\widetilde{T R P})$ can be determined from Eq. (11) using the $\alpha-$ cut of $\overparen{T R P}$ provided by either of the Eq. (12), Eq. (13) or Eq. (14) according to the position of $Q_{1}^{*}-Q_{2}^{*}$ where as $\bar{M}(\tilde{P})$ is given by Eq. (4), Eq. (6) or Eq. (8) according to the position of $Q_{2}^{*}$. In the following, we illustrate the model numerically. The benefit of product reorder instead of single order is also computed.

\section{Numerical examples}

In order to illustrate the above reordering strategy in single-period context, consider a spot seller stores woolen material for winter season. Total seasonal time can be divided into two slots each carries two months. Suppose, imprecision occurs in the customer level like 'demand is about $d$ ' and we transform this linguistic terms into triangular fuzzy numbers. Consider there are three items in stock and the system parameters for each item are presented in Table 1.

\section{Table 1}

Parametric values for different items

\begin{tabular}{cccccc}
\hline Items & $c$ & $p$ & $s$ & $h$ & Demand for whole season \\
\hline 1 & 8 & 12 & 4 & 2 & $(2100,2500,2900)$ \\
2 & 9 & 13 & 4 & 2 & $(1300,1700,2100)$ \\
3 & 10 & 14 & 4 & 2 & $(1100,1300,1500)$ \\
\hline
\end{tabular}

The choice of slot-1's demand $\widetilde{D}_{1}$ from the whole single-period's demand should be a certain percent of $\widetilde{D}(\theta \%$ say). But such a selection may not be unique from expert's point of view. It may vary from expert to expert like " $50 \%$ of $\widetilde{D}$ ", " $60 \%$ of $\widetilde{D}$ ", etc. Suppose, out of 25 experts 3 have been " $45 \%$ ", 5 "50\%", 7 " $55 \%$ ", 10 " $60 \%$ " and 5 " $65 \%$ ". In this case, the final choice of slot-1's demand can be derived by taking the fuzzy expectation of these fuzzy observations using the notion of FRV. Table 2 depicts the selection of products demand during the slots. Results for different items are presented in Table 3. The percentage increase in expected profit (PIEP) compares the benefit of ordering twice instead of one, and is computed as PIEP $=100 \times \frac{\bar{M}_{\text {re-order }}-\bar{M}_{\text {one-order }}}{\bar{M}_{\text {one-order }}}$.

Table 2

Demand information

\begin{tabular}{cll}
\hline Items & \multicolumn{1}{c}{ During slot 1} & \multicolumn{1}{c}{ During slot 2 } \\
\hline 1 & $(1423.80,1695.00,1966.20)$ & $(676.20,805.00,933.80)$ \\
2 & $(881.40,1152.60,1423.80)$ & $(418.60,547.40,676.20)$ \\
3 & $(745.80,881.40,1017.00)$ & $(354.20,418.60,483.00)$ \\
\hline
\end{tabular}


Table 3

Comparison between one order case and two order case

\begin{tabular}{lllllllll}
\hline \multicolumn{7}{c}{ One order case } & \multicolumn{2}{l}{ Two order case } \\
\hline Items & $Q^{*}$ & $\bar{M}_{\text {one-order }}$ & \multicolumn{2}{c}{$Q_{2}^{*}$} & $\bar{M}\left(\tilde{Q}_{2}^{\text {opt }}\right)$ & $Q_{1}^{*}+\bar{M}\left(\tilde{Q}_{2}^{\text {opt }}\right)$ & $\bar{M}_{\text {re-order }}$ & PIEP \\
\hline 1 & 2464 & 9118.73 & 793.73 & 522.30 & 2488.50 & 9716.23 & $6.55 \%$ \\
2 & 1658 & 5870.50 & 534.00 & 262.81 & 1686.61 & 6500.71 & $9.69 \%$ \\
3 & 1276 & 4712.34 & 409.19 & 273.59 & 1290.59 & 5043.07 & $7.01 \%$ \\
\hline
\end{tabular}

\section{Conclusion}

This paper considered a situation in which the single-period 'newsboy type' product may be ordered twice during a period. It is supposed that the single-period problem operates under uncertainty in customer demand, which is described by imprecise terms and modeled by fuzzy sets. The reordering policy adopted here is quite relevant with real business environment. As most of the shopkeepers' store multi items in their inventory, which require more storage space as well as more initial investment, our policy reduces both the space problem and the initial investment. In this case, the DM also has the opportunity to reduce the ordering policy later in the season if the realized demand in slot-1 is low. The strategy gives both DM's achievement and customer satisfaction. The proposed model can be extended to a case in which the purchasing cost during the mid-season may differ from the initial procurement cost.

\section{Acknowledgements}

The author would like to thank to anonymous referees for their valuable and constructive comments that have led to a significant improvement in the original paper.

\section{References}

Bector, R. C., \& Chandra, S. (2005). Fuzzy mathematical programming and fuzzy matrix games. New York: Springer.

Carlsson, C. \& Fuller, R. (2001). On possibilistic mean value and variance of fuzzy numbers. Fuzzy Sets and Systems, 122, 315-326.

Chung, C. S. \& Flynn, J. (2001). A newsboy problem with reactive production. Computers and Operations Research, 28, 751-765.

Dubois, D. \& Prade, H. (1987), The mean value of a fuzzy number. Fuzzy Sets and Systems, 24, 279300.

Dutta, P., Chakraborty, D. \& Roy, A.R. (2007). An inventory model for single period products with reordering strategy under fuzzy demand. Computers and Mathematics with Applications, 53, $1502-1517$.

Hadley, G. \& Whiten, T.M. (1963). Analysis of Inventory Systems, Prentice-Hall, Englewood Cliffs, NJ.

Khouja, M. (1999). The single-period (news-vender) inventory problem: a literature review and suggestions for future research. Omega, 27, 537-553.

Liou, T.-S. \& Wang, M.-J. (1992). Ranking fuzzy numbers with integral value. Fuzzy Sets and Systems, 50, 247-255.

Lau, H. S. \& Lau, A. H. L. (1997). Reordering strategies for a newsboy-type product. European Journal of Operational Research, 103, 557-572.

Lau, A. H. L. \& Lau, H. S. (1998). Decision models for single-period products with two ordering opportunities. International Journal of Production Economics, 55, 57-70.

Luhandjula, M.K. (2004). Fuzzy random variable: A mathematical tool for combining randomness and fuzziness, The Journal of Fuzzy Mathematics, 12, 755-764.

Puri, M.L. \& Ralescu, D.A. (1986). Fuzzy Random Variables. Journal of Mathematical Analysis and Applications, 114, 409-422. 